\title{
Properties of solid hydrogen doped by heavy atomic and molecular impurities
}

\author{
N.N. Galtsov, A.I. Prokhvatilov, G.N. Shcherbakov, and M.A. Strzhemechny \\ B. Verkin Institute for Low Temperature Physics and Engineering \\ of the National Academy of Sciences of Ukraine, 47 Lenin Ave., Kharkov 61103, Ukraine \\ E-mail: galtsov@ilt.kharkov.ua
}

\begin{abstract}
Using powder x-ray diffraction we studied the structural characteristics of normal and para hydrogen crystals doped with $\mathrm{Ar}, \mathrm{Kr}, \mathrm{N}_{2}$, and $\mathrm{O}_{2}$ impurities over the range from $5 \mathrm{~K}$ to the melting point of the hydrogen matrix. It has been established that in spite of very low solubility of the dopants in solid hydrogen, these impurities appreciably affect the structural characteristics. In particular, only nitrogen impurities do not change the molar volume of the matrix, the other three make the matrix expand. The Ar and $\mathrm{Kr}$ impurities also change the $c / a$ ratio of the hcp matrix. The fact that both $\mathrm{Ar}$ and $\mathrm{O}_{2}$ have smaller molar volumes than hydrogen may be treated as evidence that these impurities form van der Waals complexes with the hydrogen lattice environment.
\end{abstract}

PACS: $67.80 . \mathrm{Mg}, \mathbf{6 7 . 9 0 . + z}$

Solid mixtures of hydrogen with rare gas and simple molecular species are interesting for several reasons. At high pressures, some of such mixtures can form stoichiometric solid-state compounds, like $\operatorname{Ar}\left(\mathrm{H}_{2}\right)_{2}$ [1] or hydrogen-methane ordered alloys [2]. At low pressures, hydrogen-containing alloys with smaller molecular (atomic) species can be expected to form random binary systems that would in many aspects resemble helium-impurity gels [3,4]. Quench condensed $\mathrm{Ar}-\mathrm{H}_{2}$ mixtures at sufficiently high $\mathrm{H}_{2}$ contents in the source gas show many properties that could be treated as pertaining to gels of that kind [5]. In strongly diluted $\mathrm{H}_{2}$-based mixtures one can expect the formation of van der Waals (VdW) complexes, loosely bound to the crystal environment due to quantum-crystal effects. Evidence of such VdW complexes has been obtained by x-ray diffraction on Ne-doped para hydrogen [6]. Similar results have been obtained for other neon-doped hydrogen matrices (normal $\mathrm{H}_{2}$ and $\mathrm{D}_{2}$, ortho deuterium) [7]. In all those crystals, certain structural characteristics behaved in an unusual way, in particular, the reflection attributable to hcp hydrogen grew considerably in intensity, the molar volume increased upon doping against natural expectations, the hcp lattice flattened on doping (the $c / a$ ratio decreased). Neon impurities in solid hydrogen cause a few effects that could be explained only under the assumption that VdW complexes are present in the diluted alloys. These finding are an unusual low-temperature anomaly in the heat capacity [8], a decrease in the thermal resistance of Ne-doped alloys (instead of an expected increase) compared to pure hydrogen [9], an acceleration of quantum diffusion caused by Ne doping [10], and some others.

Behavior of atoms and smaller molecules in solid hydrogen is important in view of the recent idea of using para hydrogen as the isolation matrix material [11]. On the one hand, effect of the quantum-crystal nature of solid hydrogen on optical spectra still remains an open issue. On the other hand, the rotational dynamics of molecular impurities differs essentially in classical rare gas and quantum (hydrogen) matrices [12-16]. Presumably, the solid hydrogen matrix is softer, interacting less with the impurity embedded there to. But in classical matrices, impurity molecules (provided they do not interact) rotate quite freely down to very low temperatures. By shear contrast to quite reasonable expectations, rotation of impurity molecules in a quantum-crystal matrix is substantially hindered and even locked into a librational state along particular crystallographic directions [16]. This fact can be easily explained by the extreme compliability. Thus, it was shown [15] that a $\mathrm{SF}_{6}$ molecule in a helium matrix has a «coat» of 22 to $24 \mathrm{He}$ atoms so that rotation is greatly hindered even in the superfluid phase of helium.

There is another issue in the physics of dilute impurities in various matrices, which can be directly solved 
with the aid of diffraction methods. This issue is the changes in the molar volume of the matrix material and the relevant displacement of the closer crystal shells, necessary to be known for crystal-field evaluations and corrections.

Here we report effects of heavier atomic ( $\mathrm{Ar}$ and $\mathrm{Kr})$ as well as molecular $\left(\mathrm{N}_{2}\right.$ and $\left.\mathrm{O}_{2}\right)$ impurities on the structural characteristics of the quantum crystals of para and normal hydrogen. To facilitate understanding of the experimental findings for $\mathrm{H}_{2}$-based binary systems we give in Table the basic molecular and other parameters of the species involved.

\section{Experimental}

These studies were performed on a powder x-ray diffractometer DRON-3M equipped with a liquid-helium cryostat in the $\mathrm{Cu} K_{\alpha}$ radiation. Diffractometer control as well as data collection and processing were done using a PC. The sample were grown by quench condensation of gas mixtures of known composition directly to the solid phase onto a flat copper substrate at a temperature of $5 \mathrm{~K}$. The polycrystalline samples were typically $0.1 \mathrm{~mm}$ thick with grain sizes within $10^{-4}-10^{-5} \mathrm{~cm}$. The purity of all the source gases was not worse that $99.9 \%$. The source parahydrogen had an ortho fraction of $0.23 \%$, which is an equilibrium value for liquid-hydrogen temperature. The concentration of the impurity species in the gas mixtures was varied from $0.05 \%$ to $5 \%$ for $\mathrm{Ar}, 1 \%$ to $10 \%$ for $\mathrm{Kr}$ and $\mathrm{N}_{2}$, and from $1 \%$ to $20 \%$ for $\mathrm{O}_{2}$. The error of the impurity fraction in the gas sample was $5 \%$ of the total amount of the impurity in the gas. X-ray examination was carried out from $5 \mathrm{~K}$ up to the melting point of the hydrogen matrix. The temperature was stabilized to within $\pm 0.05 \mathrm{~K}$ at every measurement point. Because of a partial overlap of certain reflections from the hydrogen matrix and the impurity solid, the resulting lattice parameter error was larger than for pure cryocrystals but did not exceed $\pm 0.04 \%$. If should be noted that, in contrast to what we had on neon-doped hydrogen and deuterium [7], condensation by small spurts (the pressure drop in the mixing chamber being 2-3 $\mathrm{mm} \mathrm{Hg}$ ) of the mixtures with heavier impurities yielded, as expected, strongly stressed samples. This was evidenced from the absence of most of the reflections, while the observed reflection (as a rule, the 002 ones) were broad. To remove stresses, such samples were annealed for 1 to 1.5 hours at a temperature 2 to $3 \mathrm{~K}$ below the melting point.

After annealing all the reflections appear in x-ray patterns with the intensity ratios close to normal and the line width typical of mixtures. We think that quench condensation onto a substrate at $5 \mathrm{~K}$ yields samples with a large amount of lattice defects, finer than usual crystallites, and stresses. The high temperature annealing not only removes stresses due to fast crystallization and cooldown but also promotes a homogenization of the impurity distribution. This argumentation is corroborated by the results of experiments with samples grown on the same $5 \mathrm{~K}$ substrate but at a twice as fast rate (with pressure drops of 5-7 $\mathrm{Hg} \mathrm{mm}$ ). Under these conditions, the condensate surface was momentarily heated up to the melting point, producing immediately an equilibrium sample so that subsequent annealing did not change the diffraction pattern.

\section{Results and discussions}

As the impurity, two type species have been chosen, considerably differing in molecular parameters from one another and from the hydrogen matrix (see Table). This, in particular, concerns the LennardJones parameters and the Debye temperatures. The molar volume differences between impurity and matrix were such that doping of the $\mathrm{H}_{2}$ crystal would result in dilatations of opposite signs. Thus, judging from molar volumes (Table) of pure solids, argon and oxygen impurities were expected to contract the hydrogen lattice whereas krypton and nitrogen, to expand.

Table

The relevant physical properties of $n \mathrm{H}_{2}, p \mathrm{H}_{2}$ as well as impurities $\mathrm{Ar}, \mathrm{Kr}, \mathrm{N}_{2}$, and $\mathrm{O}_{2}$

\begin{tabular}{|c|c|c|c|c|}
\hline $\begin{array}{c}\text { Sub- } \\
\text { stance }\end{array}$ & $\begin{array}{c}\text { Structure } \\
\text { at } 5 \mathrm{~K} \\
\end{array}$ & $\begin{array}{c}\text { Lattice } \\
\text { parameters, } \AA \\
\end{array}$ & $\begin{array}{c}\begin{array}{c}\text { Molar volume, } \\
\mathrm{cm}^{3} / \text { mole }\end{array} \\
\end{array}$ & $\begin{array}{c}L-J \\
\text { parameters } \\
\end{array}$ \\
\hline$n \mathrm{H}_{2}$ & $\begin{array}{c}\text { hср, } \\
P 6_{3} / m m c\end{array}$ & $\begin{array}{c}a=3.770 \\
c=6.162\end{array}$ & 22.83 & $\begin{aligned} \varepsilon & =36.7 \mathrm{~K} \\
\sigma & =2.96 \AA\end{aligned}$ \\
\hline$p \mathrm{H}_{2}$ & $\begin{array}{c}\text { hсp, } \\
P 6_{3} / m m c\end{array}$ & $\begin{array}{l}a=3.783 \\
c=6.178\end{array}$ & 23.06 & $\begin{array}{l}\varepsilon=36.7 \mathrm{~K} \\
\sigma=2.96 \AA\end{array}$ \\
\hline $\mathrm{Ne}$ & $\begin{array}{c}\text { fcc, } \\
\text { Fm } 3 m\end{array}$ & $a=4.464$ & 13.31 & $\begin{aligned} \varepsilon & =36.7 \mathrm{~K} \\
\sigma & =2.788 \AA\end{aligned}$ \\
\hline Ar & $\begin{array}{c}\text { fcc, } \\
F m 3 m\end{array}$ & $a=5.311$ & 22.415 & $\begin{aligned} \varepsilon & =119.8 \mathrm{~K} \\
\sigma & =3.405 \AA\end{aligned}$ \\
\hline $\mathrm{Kr}$ & $\begin{array}{c}\text { fcc, } \\
\text { Fm } 3 m\end{array}$ & $a=5.646$ & 26.932 & $\begin{aligned} \varepsilon & =164.0 \mathrm{~K} \\
\sigma & =3.624 \AA\end{aligned}$ \\
\hline $\mathrm{N}_{2}$ & $\begin{array}{l}\text { fcc, } \\
P a 3\end{array}$ & $a=5.649$ & 27.13 & $\begin{aligned} \varepsilon & =95.1 \mathrm{~K} \\
\sigma & =3.708 \AA\end{aligned}$ \\
\hline $\mathrm{O}_{2}$ & $\begin{array}{c}\text { monoclinic, } \\
C 2 / m\end{array}$ & $\begin{array}{l}a=5.375, \\
b=3.425, \\
c=4.242, \\
\beta=117.8^{\circ}\end{array}$ & 20.57 & $\begin{array}{l}\varepsilon=117.3 \mathrm{~K} \\
\sigma=3.817 \AA\end{array}$ \\
\hline
\end{tabular}

It should be also taken into account that the molar volumes of both molecular solids $\mathrm{N}_{2}$ and $\mathrm{O}_{2}$ are to an 

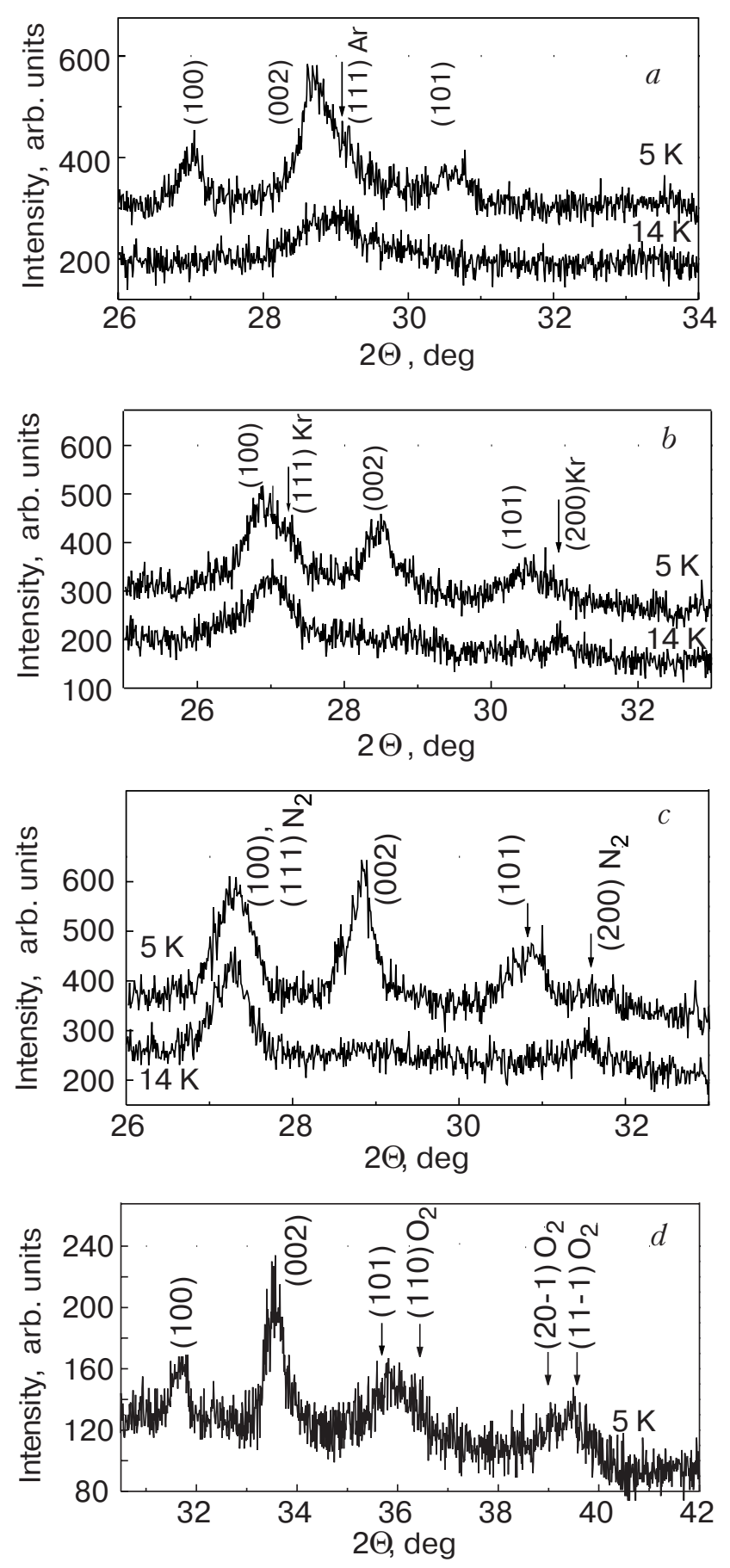

Fig. 1. Typical powder $\mathrm{x}$-ray patterns for solid mixtures: $p \mathrm{H}_{2}+1 \% \operatorname{Ar}(a), n \mathrm{H}_{2}+2 \% \mathrm{Kr}(b), p \mathrm{H}_{2}+2 \% \mathrm{~N}_{2}(c)$ for $5 \mathrm{~K}$ and $14 \mathrm{~K}, n \mathrm{H}_{2}+2 \% \mathrm{O}_{2}$ for $5 \mathrm{~K}(d)$.

appreciable extent controlled by rather strong anisotropic interactions, which tend to compress these solids. In addition, the paramagnetic impurities of oxygen can essentially affect the conversion process in the normal hydrogen crystals [20-22].

Some of preliminary results of hydrogen doped with $\mathrm{Ar}, \mathrm{Kr}$, and $\mathrm{N}_{2}$ have been reported at the 3rd Cryocrystals Conference [23]. Later we carried out a few complementing and more precise experiments on the these above-mentioned systems, in particular, using normal hydrogen as matrix, as well as repeated the entire set of measurements on oxygen-doped normal hydrogen.

When embarking on this program, we expected to find evidence of $\mathrm{VdW}$ complexes around the impurity particles. However, our analysis shown that there are no unambiguous confirmation of this hypothesis, at least within the content sensitivity (about 1\%) of our method. The x-ray patterns contained reflections only from the hcp hydrogen-rich phases (both for normal and para $\mathrm{H}_{2}$ ) and, when observable, reflections from the lattices of the respective pure substances (Fig. 1), the monoclinic lattice of $\mathrm{O}_{2}$ and fcc lattices of all other crystals. It should be remarked here that the determination of the least concentration, at which impurity-based phases used to appear, was difficult because for krypton and nitrogen impurities the (111) reflection of the respective cubic phases overlapped in part with the first (100) reflection of $\mathrm{H}_{2}$; for argon and oxygen - with second (002) and third (101) reflection of $\mathrm{H}_{2}$ matrix respectively (cf. Fig. 1, $a$ to $d$ ). However, in spite of these aggravations we have established that reflections of the pure phases of all the dopants are detectable in x-ray patterns when the normal fraction of the impurity in the source gas mixture exceed $0.5 \%$.

Typical powder x-ray patterns for hydrogen-based solid mixtures with the four impurity species are shown in Fig. 1. The position and shapes of the $\mathrm{H}_{2}$ reflections differ from those from pure normal and para hydrogen. This is might be caused by the following factors. Although the actual content (solubility) of all impurities can be substantially less than the lower concentration in gas mixtures (the equilibrium solubility of heavy gases in solid hydrogen from thermal conductivity measurements $[24,25]$ is $10^{-4}$ or less), these impurities affect perceptibly the structural characteristics of doped hydrogen. Usually, when quench depositing pure hydrogen on low temperature, it is difficult to avoid texture [26] with the close packed (001) basal layers being parallel to the substrate surface so that multiple reflections from these planes can only be seen in patterns. In the experiments reported here, even seemingly insignificant amounts of impurities (as low as $0.05 \%$ of $\mathrm{Ar}$ in the gas mix) essentially suppresses preferable epitaxial crystal growth and the intensity ratios from the hydrogen matrix is close to that from non-textured polycrystalline samples (cf. for example, Fig. 1, $a$ ). The part of the impurity component that was not dissolved in solid hydrogen aggregates into a separate phase producing corresponding reflections. Above the hydrogen melting temperature, only impurity-related reflections persist (Fig. 1). The 


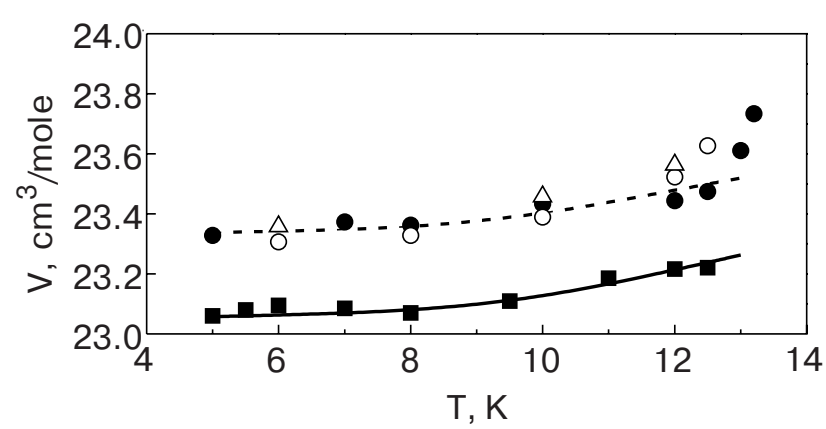

Fig. 2. Temperature dependence of the molar volume of solid para hydrogen with impurities: $0.05 \% \operatorname{Ar}(\bullet) ; 2 \% \mathrm{~N}_{2}$ (ם); pure $\mathrm{pH}_{2}$ [26] (-); 5\% $\mathrm{Ar}(\triangle) ; 2 \% \mathrm{Ne}[6]$ (- - -); $2 \% \operatorname{Ar}(\mathrm{O})$.

integrated intensity of these reflections is noticeably higher than could be expected from the nominal concentration in the gas. The width of the reflections after annealing-related hydrogen effusion is considerably (2-2.5 times) larger than usual, which suggests a high concentration of impurities $\left(\mathrm{H}_{2}\right)$ and/or lattice defects.

Before analyzing the structure data and giving our arguments, we note that, when considering a molecular impurity in a solid made up of spherical particles (like $\mathrm{H}_{2}$ ), one should use for scaling not the molar volume of the pure molecular solid (in which strong anisotropic force produce a large negative contribution) but, because in an environment of spherical particles the molecule is stripped of its anisotropic forces, $\sigma^{3}$ where $\sigma$ is the Lennard-Jones radius [27].

Argon impurities in para hydrogen increase the volume of the matrix by an amount comparable to those observed in $p \mathrm{H}_{2}-\mathrm{Ne}$ mixtures (Fig. 2) despite the slightly smaller volume in the bulk Ar compared to $p \mathrm{H}_{2}$.

Oxygen impurities also expand the normal hydrogen lattice, approximately by the same amount as krypton. There is, however, an important difference because oxygen is known to accelerate ortho-para con-

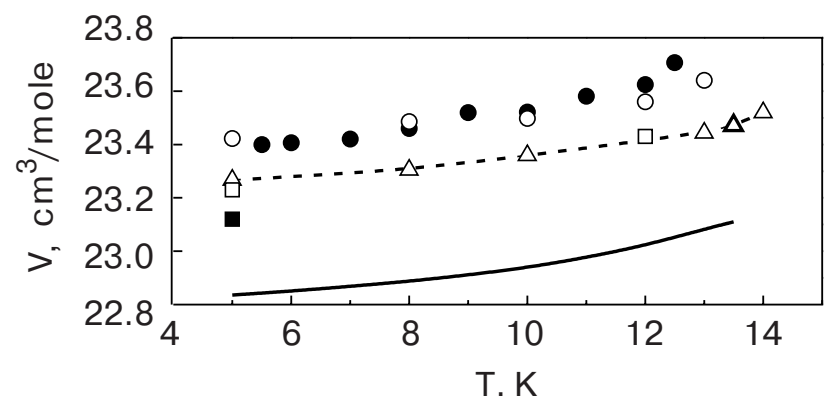

Fig. 3. Temperature dependence of molar volume of solid normal hydrogen with impurities: $1 \% \mathrm{Kr}(\bullet) ; 10 \% \mathrm{Kr}$

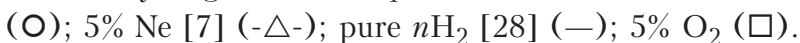

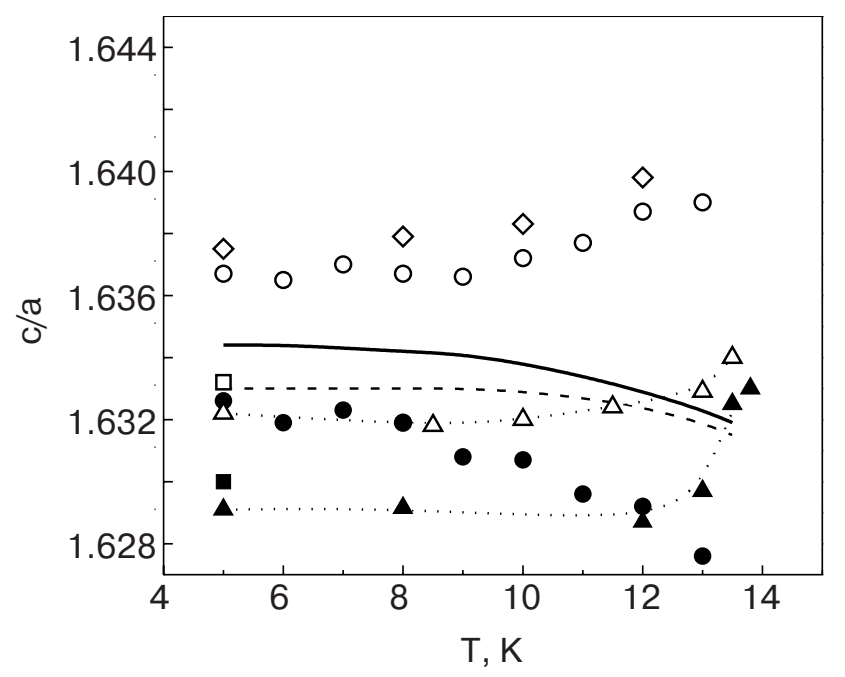

Fig. 4. Temperature dependence of the $c / a$ of solid hydrogens with impurities: $p \mathrm{H}_{2}+0.05 \% \mathrm{Ar}(\bullet) ; n \mathrm{H}_{2}+1 \%$ $\mathrm{Kr}(\mathrm{O}) ; n \mathrm{H}_{2}+5 \% \mathrm{Ne}$ [7] ( $\left.\triangle\right)$; pure $n \mathrm{H}_{2}$ [28] (-); pure $p \mathrm{H}_{2}$ [26] (- - ); $n \mathrm{H}_{2}+1 \% \mathrm{O}_{2}(\square) ; p \mathrm{H}_{2}+5 \% \mathrm{Ne}$ [6] ( $\mathbf{\Delta})$.

version so that during sample preparation and measurements oxygen impurities burn out ortho states in their closest environment and, thus, are in fact surrounded by virtually pure para hydrogen. Therefore, the net expansion effect due to $\mathrm{O}_{2}$ impurities is less pronounced compared to $\mathrm{Kr}$ impurities. Thus, the lattice expansion caused by $\mathrm{Ar}$ and $\mathrm{O}_{2}$ impurities, both of which are smaller than the size of vacant sites in hydrogen crystals, may be treated as evidence of hydrogen-based VdW complexes, similar to those presumably found in Ne-doped hydrogen.

Nitrogen apparently does not change the volume hydrogen matrix at any temperature up to melting (Fig. 2). Since Lennard-Jones radius of the bare nitrogen molecule (3.708 $\AA$ ) is close to the intermolecular distance in solid $\mathrm{H}_{2}$ (3.784 $\AA$ for para hydrogen), one can expect an «accurate» nesting for the $\mathrm{N}_{2}$ impurity in $\mathrm{H}_{2}$. Since the central $\mathrm{H}_{2}-\mathrm{H}_{2}$ interaction constant does not differ drastically from $\mathrm{H}_{2}-\mathrm{N}_{2}$ one, the almost absent effect of $\mathrm{N}_{2}$ does not seem strange.

Krypton impurities increase the molar volume of the normal hydrogen matrix (Fig. 3). The fact that the excess volume and its temperature dependence are the same for the nominal gas fractions of $1 \%$ and $10 \%$ implies that the true $\mathrm{Kr}$ concentration in solid $\mathrm{H}_{2}$ must not extend 1\%. Our previous evaluation [5] yields an upper limit value of $4 \%$, which does not contradict the above reasoning. The very fact of the positive effect of $\mathrm{Kr}$ impurities seems to be quite natural because of the larger impurity size.

Although Ar and $\mathrm{Kr}$ bring about almost equal volume changes, they deform the hexagonal $\mathrm{H}_{2}$ lattice in a different way (Fig. 4): Ar decreases and $\mathrm{Kr}$ increases 
the $c / a$ ratio and both absolute deviations increase with increasing temperature. The opposite behavior of the $c / a$ ratio (temperature independence close to melting, then a fast buildup) in $\mathrm{Ne}-\mathrm{H}_{2}$ mixtures [6] can be due to a possible destruction of $\mathrm{Ne}\left(\mathrm{H}_{2}\right)_{n}$ complexes at higher temperatures.

All the facts listed above imply that the doping of such a quantum crystal like hydrogen with heavier impurities and, especially, quantum-crystal effects in the dynamics of substitutional impurities in quantum solids cannot be described by the theory of regular solid solutions. The experimental facts reported here might serve as another stimulating arguments for theorists.

This work was supported by CRDF (grant UP2-2445-KH-02). The authors thank V.G. Manzhelii and M.I. Bagatskii for valuable discussions.

1. P. Loubeyre, R. LeToullec, and J.P. Pinceaux, Phys. Rev. Lett. 72, 1360 (1994).

2. M.S. Somayazulu, L.W. Finger, R.J. Hemley, and H.K. Mao, Science 271, 1400 (1996).

3. E.B. Gordon, L.P. Mezhov-Deglin, and O.F. Pugachev, Pisma Zh. Eksp. Teor. Fiz. 19, 103 (1974).

4. V.V. Khmelenko, S.I. Kiselev, D.M. Lee, and C.Y. Lee, Physica Scripta 102, 118 (2003).

5. M.A. Strzhemechny, N.N. Galtsov, and A.I. Prokhvatilov, Fiz. Nizk. Temp. 29, 699 (2003) [Low Temp. Phys. 29, 522 (2003)].

6. A.S. Barylnik, A.I. Prokhvatilov, M.A. Strzhemechny, and G.N. Shcherbakov, Fiz. Nizk. Temp. 19, 625 (1993) [Low Temp. Phys. 19, 447 (1993)].

7. A.S. Barylnik, A.I. Prokhvatilov, and G.N. Shcherbakov, Fiz. Nizk. Temp. 21, 788 (1995) [Low Temp. Phys. 21, 607 (1995)].

8. I.Ya. Minchina, M.I.Bagatskii, V.G. Manzhelii, and P.I. Muromtsev, Fiz. Nizk. Temp. 21, 711 (1995) [Low Temp. Phys. 21, 530 (1995)].

9. B.Ya. Gorodilov, A.I. Krivchikov, V.G. Manzhelii, and N.N. Zholonko, Fiz. Nizk. Temp. 20, 78 (1994) [Low Temp. Phys. 20, 66 (1994)].

10. M.I. Bagatskii, I.Ya. Minchina, and V.G. Manzhelii, Fiz. Nizk. Temp. 22, 52 (1996) [Low Temp. Phys. 22, 37 (1996)].

11. T. Momose and T. Shida, Bull. Chem. Soc. Jpn. 71, 1 (1998).
12. A.V. Danilychev, V.E. Bondybey, V.A. Apkarian, S. Tanaka, H. Kajihara, and S. Koda, J. Chem. Phys. 103, 4292 (1995)

13. M.E. Fajardo, S. Tam, T.L. Thompson, and M.E. Cordonnier, Chem. Phys. 189, 351 (1994).

14. T. Momose, M. Miki, T. Wakabayashi, T. Shida, M.-C. Chan, S.S. Lee, and T. Oka, J. Chem. Phys. 107, 7707 (1997)

15. M. Hartmann, R.E. Miller, J.P. Toennies, and A. Vilesov, Science 272, 1631 (1996).

16. J.Z. Li and V.A. Apkarian, J. Chem. Phys. 107, 1544 (1997)

17. Cryocrystals, B.I. Verkin and A.F. Prikhot'ko (eds.), Naukova Dumka, Kiev (1983) [in Russian].

18. Rare Gas Solids, M.L. Klein and J.A. Venables (eds.), Academic Press, London, Vol. 1 (1976).

19. V.G. Manzhelii, A.I. Prokhvatilov, I.Ya. Minchina, and L.D. Yantsevich, Handbook of Binary Solutions of Cryocrystals, Begell House, New York (1996).

20. K. Motizuki and T. Nagamiya, J. Phys. Soc. Jpn. 11, 93 (1956)

21. V. Shevtsov, A. Shcherbakov, P. Malmi, E. Ylinen, and M. Punkkinen, J. Low Temp. Phys. 104, 211 (1996).

22. V. Shevtsov, P. Malmi, E. Ylinen, and M. Punkkinen, J. Low Temp. Phys. 114, 431 (1999).

23. G.N. Shcherbakov, A. I. Prokhvatilov, M.A. Strzhemechny, and A.S. Barylnik, Abstracts of 2nd Intern. Confer. Cryocrystals and Quantum Crystals, Polanica-Zdroj, Poland (1997), p. 2.

24. B.Ya. Gorodilov, A.I. Krivchikov, V.G. Manzhelii, N.N. Zholonko, and O.A. Koroluk, Fiz. Nizk. Temp. 21, 723 (1995) [Low Temp. Phys. 21, 561 (1995)].

25. V.G. Manzhelii, B.Ya. Gorodilov, and A.I. Krivchikov, Fiz. Nizk. Temp. 22, 174 (1996) [Low Temp. Phys. 22, 131 (1996)].

26. I.N. Krupskii, A.I. Prokhvatilov, and G.N. Shcherbakov, Fiz. Nizk. Temp. 9, 84 (1983) [Sov. J. Low Temp. Phys. 9, 42 (1983)].

27. M.A. Strzhemechny, A.I. Prokhvatilov, and L.D Yantsevich, Physica B198, 267 (1994).

28. I.N. Krupskii, A.I. Prokhvatilov, and G.N. Shcherbakov, Fiz. Nizk. Temp. 9, 858 (1983) [Sov. J. Low Temp. Phys. 9, 446 (1983)]. 